\title{
Fatores relacionados à adesão ao tratamento anti-hipertensivo: fundamentação a partir da teoria de Imogene King
}

RESUMO | Objetivo: Conhecer as percepções de usuários hipertensos diante do tratamento anti-hipertensivo, relacionandoas com os conceitos do sistema interpessoal da Teoria do Alcance de Metas de Imogene King. Método: Estudo descritivo e exploratório, com abordagem qualitativa, realizado com 10 usuários hipertensos. Foram realizadas entrevistas semiestruturadas para a coleta dos dados e utilizou-se a Análise de Conteúdo Temática proposta por Minayo para análise do material. Resultados: Foi possível identificar dois eixos temáticos: Viver com Hipertensão Arterial Sistêmica; Dificuldades diante da adesão ao tratamento anti-hipertensivo. Conclusão: A necessidade de uso contínuo da medicação, mudanças no cotidiano dos indivíduos e o desconhecimento em relação à patologia, são fatores que podem influenciar a adesão ao tratamento anti-hipertensivo. Ressalta-se que a relação entre o enfermeiro e o paciente é compreendida como aspecto que contribui para a terapêutica.

Palavras-chaves: Hipertensão Arterial; Adesão ao tratamento; Atenção Primária a Saúde.

\begin{abstract}
Objective: To know the perceptions of hypertensive users regarding antihypertensive treatment, relating them to the concepts of the interpersonal system of the Theory of Goal Achievement by Imogene King. Method: Descriptive and exploratory study, with a qualitative approach, carried out with 10 hypertensive users. Semi-structured interviews were conducted to collect the data and the Thematic Content Analysis proposed by Minayo was used to analyze the material. Results: It was possible to identify two thematic axes: Living with Systemic Arterial Hypertension; Difficulties with adherence to antihypertensive treatment. Conclusion: The need for continuous use of medication, changes in the daily lives of individuals and the lack of knowledge about the pathology, are factors that can influence adherence to antihypertensive treatment. It is noteworthy that the relationship between the nurse and the patient is understood as an aspect that contributes to therapy.
\end{abstract}

Keywords: Hypertension; Adherence to treatment; Primary Health Care.

RESUMEN | Objetivo: Conocer las percepciones de los usuarios hipertensos sobre el tratamiento antihipertensivo, relacionándolas con los conceptos del sistema interpersonal de la Teoría del Logro de Metas de Imogene King. Método: Estudio descriptivo y exploratorio, con abordaje cualitativo, realizado con 10 usuarios hipertensos. Se realizaron entrevistas semiestructuradas para recolectar los datos y se utilizó el Análisis de Contenido Temático propuesto por Minayo para analizar el material. Resultados: Fue posible identificar dos ejes temáticos: Vivir con Hipertensión Arterial Sistémica; Dificultades para la adherencia al tratamiento antihipertensivo. Conclusión: La necesidad de uso continuo de medicamentos, los cambios en la vida diaria de los individuos y el desconocimiento de la patología, son factores que pueden influir en la adherencia al tratamiento antihipertensivo. Es de destacar que la relación entre la enfermera y el paciente se entiende como un aspecto que contribuye a la terapia.

Palabras claves: Hipertensión; Adherencia al tratamiento; Primeros auxilios.

\section{Deiziane Serafim de Oliveira}

Enfermeira graduada pela Faculdade de Enfermagem Nova Esperança.

ORCID: 0000-0003-4090-1447

\section{Bárbara Cristina da Silva Oliveira}

Enfermeira graduada pela Faculdade de Enfermagem Nova Esperança.

ORCID: 0000-0002-4058-5117

\section{Salmana Rianne Pereira Alves}

Enfermeira. Mestre pelo Programa de Pós- graduação em Saúde da Família, pela Faculdade de Enfermagem Nova Esperança.

ORCID: 0000-0002-4472-2289

\section{Valdicléia da Silva Ferreira Torres}

Enfermeira. Mestre em Enfermagem pelo Programa de Pós-graduação em Enfermagem da UFPB.

ORCID: 0000-0002-3974-7123

\section{Regina Célia de Oliveira}

Enfermeira. Pós-Doutora, Universidade de Pernambuco/UPE.

ORCID: 0000-0002-6559-5872

\section{Camila Abrantes Cordeiro Morais}

Enfermeira. Doutoranda em Enfermagem pelo Programa Associado de Pós-graduação em Enfermagem da UPE-UEPB.

ORCID: 0000-0003-3780-9340

Recebido em: 28/02/2021

Aprovado em: 15/03/2021

INTRODUÇão

m 2013, cerca de $72,6 \%$ das
mortes no Brasil foram atribuídas
às Doenças Crônicas Não Transmissíveis (DCNT) que, além de causar ele- vado número de mortes prematuras, declínio na qualidade de vida com alto grau de limitação e incapacidade, também acarretam importante impacto econômico para o indivíduo, família e sociedade. ${ }^{(1,2)}$

Dentre as DCNT, as doenças cardiovasculares representam a principal causa de morte no Brasil, com mais de $30 \%$ de óbitos registrados. São cerca de 43 óbitos por hora, ou seja, 1 morte a cada $90 \mathrm{mi}-$ nutos $^{(2)}$. No acompanhamento dos indivíduos com DCNT, a Atenção Primária a Saúde (APS) desempenha papel essencial para o sucesso do controle e tratamento dessas doenças, uma vez que, desenvolvem ações voltadas para a prevenção dos agravos, tratamento, promoção e reabilitação da saúde. ${ }^{(3)}$ 
Um estudo realizado com o objetivo de identificar as necessidades de aprendizado em saúde de pacientes hipertensos hospitalizados, demonstrou que os pacientes possuíam elevado grau de dependência e incapacidades graves, além de déficit de conhecimento relacionado à patologia, levando à não adesão ao tratamento, potencializando a ocorrência de complicações decorrentes da doença. ${ }^{(4)}$

É importante que os profissionais de saúde, em especial a equipe de enfermagem, mantenham uma constante interação no cuidado a esses indivíduos, estimulando a adesão ao processo terapêutico, auxiliando a compreensão da necessidade de adotar alterações no seu estilo de vida. ${ }^{(5)}$

Considerando que a inter-relação entre profissional e paciente ocorre através do estabelecimento da interação entre ambos, o Modelo Conceitual de Sistemas Abertos (MCSA), proposto por Imogene King, em 1981, enfatiza que o indivíduo está inserido em três sistemas interativos: o pessoal, o interpessoal, e o social. A Teoria de King pode ser aplicada junto ao usuário hipertenso, especialmente aqueles que não apresentam uma adequada adesão ao tratamento, em virtude do estabelecimento de metas entre o enfermeiro e o paciente, no intuito de enfrentar dificuldades relacionadas ao cumprimento do tratamento proposto. ${ }^{(6)}$

Nessa perspectiva, considerando o caráter assintomático e crônico da hipertensão arterial, os diversos desafios que envolvem as mudanças ocasionadas pelo diagnóstico e a importância do estabelecimento da interação entre o profissional de saúde e o paciente, surgiu o seguinte questionamento: Que fatores podem influenciar à adesão ao tratamento anti-hipertensivo? A relação interpessoal estabelecida entre o enfermeiro e o usuário hipertenso pode contribuir para a adesão ao tratamento anti-hipertensivo?. Esta pesquisa apresenta como objetivo conhecer as percepções de usuários hipertensos diante do tratamento anti-hipertensi- vo, relacionando-as com os conceitos do sistema interpessoal da Teoria do Alcance de Metas de Imogene King.

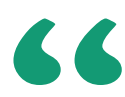

\section{É importante que os profissionais de saúde, em} especial a equipe de enfermagem, mantenham uma constante interação no cuidado a esses indivíduos, estimulando a adesão ao processo terapêutico, auxiliando a compreensão da necessidade de adotar alterações no seu estilo de vida.

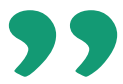

MÉTODO

Trata-se de uma pesquisa de caráter exploratório e descritivo, com abordagem qualitativa, realizada em uma Uni- dade de Saúde da Família (USF) localizada no município de João Pessoa-PB. A população foi composta pelos usuários hipertensos cadastrados na referida USF, sendo a amostra constituída por 10 usuários que atenderam os seguintes critérios de inclusão: estar regularmente cadastrado e acompanhado na USF, ter idade superior ou igual a 18 anos, de ambos os sexos e ter diagnóstico de hipertensão arterial há pelo menos um ano. Foram excluídos aqueles com déficit cognitivo e com dificuldades passíveis de inviabilizar a comunicação e as respostas ao instrumento, assim como os que não estavam presentes na instituição no momento da coleta de dados.

Para viabilizar a coleta dos dados, foi utilizado um roteiro de entrevista semiestruturado, elaborado pelos pesquisadores, a partir de um roteiro com questões pertinentes ao objetivo do estudo. A entrevista foi gravada por meio da utilização de um sistema de gravação Mp4, com a anuência dos participantes.

A coleta de dados ocorreu no período de agosto a setembro de 2019. Foi apresentado aos participantes, o termo de consentimento livre e esclarecido (TCLE), assegurando-os anonimato sobre seu depoimento e autonomia de permanecer na pesquisa ou não. Os participantes também foram esclarecidos sobre o objetivo da pesquisa As entrevistas foram realizadas na própria instituição, com horário previamente agendado de acordo com a disponibilidade dos participantes, com duração média de 20 minutos.

A análise dos dados ocorreu através da Análise de Conteúdo Temática proposta por Minayo ${ }^{(7)}$ que compreende três etapas: pré-análise, exploração do material e tratamento dos resultados obtidos. Foram codificadas as palavras-chave e frases com os mesmos sentidos, as quais possibilitaram a construção das categorias temáticas.

A fim de preservar o anonimato dos participantes, os depoimentos foram identificados por nomes de "Orquídeas". Os aspectos éticos foram respeitados e 
a pesquisa seguiu as recomendações estabelecidas pela resolução 466/12 do Conselho Nacional de Saúde para pesquisas realizadas com seres humanos, sendo aprovado pelo Comitê de Ética e Pesquisa (CEP) com $n^{\circ}$ do CAAE: 09075919.0.0000.5179.

\section{RESULTADOS}

Observou-se que a maioria dos participantes era do sexo feminino (90\%) e apresentavam faixa etária entre 30 à 77 anos. Quanto a escolaridade, 60\% dos entrevistados possuía ensino fundamental completo, $2 \%$ tinham o fundamental incompleto e $3 \%$ eram analfabetos. No que diz respeito ao estado civil, 60\% eram casados. A partir da análise das informações empíricas advindas das entrevistas, emergiram duas categorias temáticas: Viver com Hipertensão Arterial Sistêmica e Desafios diante da adesão ao tratamento anti-hipertensivo.

Viver com Hipertensão Arterial Sistêmica

Ao serem questionados sobre a repercussão da descoberta da hipertensão arterial, os participantes revelaram a presença de sentimentos como o medo diante do diagnóstico, angústia e preocupação, conforme observado a seguir:

[...] fui diagnosticada há 3 anos, quando eu descobri eu tive medo [...] tive aquele choque [...] (Orquídea Lilás)

[...] quando descobri que estava com mais essa doença fiquei com muito medo [...] (Orquídea Violacea)

[...] tenho hipertensão há 5 anos [...] fiquei muito preocupada, não sabia nem o que era [...] (Orquídea Borboleta)

Ante o exposto, é importante conhecer as percepções de usuários hipertensos diante da doença e do tratamento. Aspectos relacionados as manifestações clínicas, autocuidado e déficit de conhecimento acerca da patologia foram desta- cados pelos participantes, como observado nas seguintes falas:

[...] viver com hipertensão é péssimo, só vivo com dor de cabeça, indisposta, cansada, tenho coragem pra nada, desde que descobri que sou assim [...] (Orquídea Violácea)

[...] minha pressão só vive alta, hoje mesmo tá em vinte, fico bem preocupada [...] (Orquídea Amarela)

[...] depois eu entendi que só era cuidar que não ia se agravar, eu me preocupo, pois se eu não me cuidar posso piorar e ter um problema muito maior no coração [...] (Orquídea Lilás)

Desafios diante da adesão ao tratamento anti-hipertensivo

Em relação ao tratamento anti-hipertensivo, os usuários relataram dificuldades para a adesão terapêutica, destacando: o não cumprimento das orientações, as mudanças no estilo de vida, uso contínuo da medicação, o acesso ao medicamento, o déficit de conhecimento sobre a doença e a necessidade de acompanhamento contínuo dos valores pressóricos conforme destacado a seguir:

[...] não tenho orientação nenhuma de minha doença, nem sei bem como é a doença [...] (Orquídea Violácea)

[...] a dificuldade é a pessoa não poder comer o que quer e tomar num sei quantos remédios é muito estressante [...] (Orquídea Lilás) [...] muitas vezes falta o medicamento, o que atrapalha pois tenho que comprar [...] (Orquídea Borboleta)

[...] tenho dificuldade pois tomo três remédios e me perco nos horários (Orquídea Borboleta)

[...] a única coisa chata que acho é a medicação todo dia, ficar vendo se a pressão ta alta ou baixa [...] (Orquídea Pipoca)
[...] o que me incomoda é o fato que preciso ficar o tempo todo tomando medicamento, isso é muito ruim, eu sei que eu preciso, aí tomo, já tomava a insulina, agora chegou mais coisa [...] (Orquídea Iracema)

Considerando a importância da relação interpessoal entre os profissionais de saúde, o enfermeiro, paciente e o atendimento na instituição de saúde, foi possível perceber o impacto positivo da interação entre enfermeiro e paciente, de modo a contribuir no tratamento:

[...] O meu relacionamento com a enfermeira é maravilhoso, não só pela amizade, mais trata todo mundo bem, é muito humana, não tenho do que reclamar [...] (Orquídea Branca).

[...] Eu adoro ela, me acolheu super bem quando cheguei aqui, quando descobri que era hipertensa, e ela me orientou muito sobre o que eu devia fazer [...] (Orquídea Cereja)

[...] A enfermeira sempre pergunta como eu tô, se estou me cuidando direitinho, isso pra mim faz toda a diferença [...] Se ela não me ajudasse tanto eu nem tava tomando os remédios. [..] nunca me deixou com duvida do que preciso fazer[...] (Orquídea Borboleta)

É notório que a participação e entendimento do paciente tratamento no seu plano de cuidado para a realização de ações preventivas de maneira conjunta com o profissional de saúde e a consequente resposta obtida por uma busca ativa e consciente de recursos pode ser a chave para se alcançar o resultado esperado.

\section{DISCUSSÃO}

A vida cotidiana do usuário com hi- 
pertensão é explícita no (des)cuidado de si com a doença crônica e os sentimentos de tristeza e ansiedade são expressos muitas vezes pela falta de controle da doença. Sendo assim, vidando prevenir o risco de complicações, há necessidade de orientações sobre o uso de medicamentos e o empoderamento do paciente crônico para o autocuidado. ${ }^{(8)}$

A ideia de autonomia do sujeito relacionado à sua saúde, no que tange à adesão ao tratamento anti-hipertensivo, remete a valorização do paciente, quanto ao respeito e à visão aos seus limites, de modo que o plano terapêutico seja estabelecido de acordo com a individualidade de cada um. Dessa forma, esta adesão representa um processo complexo que pode ser influenciado por diversos fatores como: a repercussão da doença na vida do usuário, acesso a instituição de saúde, planejamento terapêutico, o relacionamento paciente / profissional, além da cultura e da crença do paciente. ${ }^{(9)}$

A assistência de enfermagem tem um papel transformador acerca das orientações da doença do paciente dirigida a partir de um entendimento da complexidade da doença, já que esta envolve aspectos socioculturais que requerem dos profissionais uma abordagem integral, humanizada e que atenda as dimensões biopsicossociais do sujeito. Nesse sentido, o cuidado de enfermagem deve priorizar as ações de prevenção e promoção da saúde, orientando essas pessoas sobre a importância da prática de atividades físicas, uso regular da medicação e alimentação saudável. ${ }^{(10)}$

O enfermeiro tem papel indispensável nas orientações ao paciente a acerca de sua doença, assim como um olhar clinico para os fatores de risco, proporcionando a redução dos possíveis agravos ocasionados pela doença. Deve-se atentar para a história clínica do paciente, investigando a ocorrência de lesões em órgãos alvos e à observação se há incapacidade do paciente para realizar o seu autocuidado, permitindo assim, identificar a qualidade da assistência prestada, além de propor- cionar a reformulação de estratégias para evitar possíveis complicações. ${ }^{(11)}$

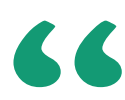

Fatores como a diversidade dos fármacos e a organização quanto à dosagem e horário podem dificultar a adesão ao tratamento anti-hipertensivo, sendo necessário reforçar as informações acerca da importância de realizar o tratamento se maneira contínua, mesmo na ausência de sintomas.
A falta de medicamentos na unidade de saúde pode atuar como uma grande barreira, pois muitos pacientes não têm a condição financeira para adquirir as medicações, fato este que torna um grande aliado no que diz respeito ao abandono do tratamento anti-hipertensivo ${ }^{(2)}$. Outro fator destacado é a presença do uso de medicamentos de maneira contínua, assim como a utilização vários fármacos para o tratamento da patologia e comorbidades associadas que podem interferir diretamente na adesão ao tratamento medicamentoso. ${ }^{(12)}$

Fatores como a diversidade dos fármacos e a organização quanto à dosagem e horário podem dificultar a adesão ao tratamento anti-hipertensivo, sendo necessário reforçar as informações acerca da importância de realizar o tratamento se maneira contínua, mesmo na ausência de sintomas. ${ }^{(13)}$

O enfermeiro como protagonista da APS, deve estar preparado para estabelecer uma comunicação efetiva que sirva como instrumento, tanto no processo de promoção a saúde, como nos cuidados assistenciais, contornando os seus desafios na construção de vinculo do serviço com o usuário. A relação enfermeiro-paciente ocorre principalmente de forma interpessoal e constitui uma ferramenta favorável para o desenvolvimento da prática Inter profissional, possibilitando o melhor acolhimento para o paciente, tendo como base a comunicação que é essencial para a promoção da saúde do usuário. ${ }^{(14)}$

$\mathrm{Na}$ interação enfermeiro-cliente pressupõe-se que: as percepções (objetivos, necessidades e valores) da enfermeira e do cliente influenciam o processo de interação; as metas, as necessidades e os valores da enfermeira e do cliente influenciam o processo de interação; os clientes têm o direito de conhecimento sobre eles mesmos e de participar das decisões que influenciam sua vida. ${ }^{(15)}$

De acordo com a Teoria de Imogene King, o papel da enfermagem pode ser definido como uma interação entre dois 
ou mais indivíduos em uma situação na qual o profissional de enfermagem usa o conhecimento, as habilidades e os valores identificados como pertinentes à identificação de metas em cada situação e para ajudar os indivíduos a alcançá-las. ${ }^{(16)}$

Tal interação ocorre a partir de estímulo para interação recíproca no estabelecimento de um sistema interpessoal positivo, sendo influenciada pelo desempenho dos papéis individuais. Faz-se necessário investigar as percepções das interações entre as pessoas, as informações comunicativas e os valores das transações nas situações de enfermagem. O conhecimento das interações humanas também auxilia a reunir informações precisas e relevantes sobre o cliente. ${ }^{(17)}$

O desconhecimento da população acerca do papel do enfermeiro na UBS pode influenciar na organização seu processo do cuidar, dificultando a implementação de ações de promoção à saúde ${ }^{(18)}$. Sendo cultural os usuários acreditarem que somente a consulta médica é resolutiva, estudos apontam que os usuários costu- mam ficar "satisfeitos" quando saem da unidade de saúde com solicitação de exame ou medicamentos prescritos, e que as orientações de cuidados e autocuidado são pouco valorizadas. ${ }^{(14)}$

A Teoria de Imogene King enfatiza a presença da comunicação como significado de intercâmbio entre pensamentos e opiniões entre os indivíduos. É considerado um processo pelo qual há troca de informações entre uma pessoa e outra, sendo esta transmitida diretamente ou não. Esta informação pode ocorrer de modo verbal, pelo contado direto, ou não-verbal, por meio de gestos, expressões faciais, ações e postura de ouvir e sentir. ${ }^{(6)}$

Sendo assim, a comunicação é fundamental para o cuidado de enfermagem, posto este exige habilidade e conhecimento da comunicação para reunir informações precisas sobre o comportamento do ser humano. Deste modo, a enfermeira tem a responsabilidade de manter uma comunicação aberta com o cliente para mutuamente traçarem metas e alcançá-las. ${ }^{(20)}$

\section{CONCLUSÃO}

Este estudo permitiu conhecer os fatores que influenciam a adesão ao tratamento anti-hipertensivo, destacando os desafios relacionados ao cumprimento adequado das medicações, às necessidades de mudanças no cotidiano dos indivíduos, além do desconhecimento em relação à patologia. Ressalta-se que, diante dos resultados elencados, a relação entre o enfermeiro e o paciente é compreendida como fator que contribui para o alcance da adesão ao tratamento.

As limitações do estudo estão relacionadas ao quantitativo de participantes entrevistados, bem como a realização da pesquisa em uma única instituição de saúde. Sendo assim, faz-se necessário novas pesquisas na área, com o intuito de ampliar as ações e metas que possam contribuir para políticas de saúde que possam promover ações educativas que atendam as demandas dos usuários, assim como discussões acerca do papel do enfermeiro no âmbito da Atenção Primária, junto a essa população.

\section{Referências}

1. World Health Organization. Adherence to long term therapies: evidence for action [internet]. Geneva (CH): WHO; 2013.

2. Gewehr DM, Bandeira VAC, Gelatti GT, Colet CF, Oliveira KR. Adesão ao tratamento farmacológico da hipertensão arterial na Atenção Primária à Saúde. Saúde Debate. 2018; 42(116):179-90.

3. Ferreira SRS, Périco LAD, Dias VRFG. A complexidade do trabalho do enfermeiro na Atenção Primária à Saúde. Rev Bras Enferm [Internet] 2018;71(supl1):752-7.

4. Souza NPG et al. Adoecimento por hipertensão arterial e Diabetes Mellitus: concepções de um grupo de pacientes hospitalizados. Rev enferm UERJ, Rio de Janeiro, 2015; 23(1):52-7.

5. Lima MS, Monteiro LD, Nogueira LSS et al. Cuidado de enfermagem à família de pacientes internados em Unidade de terapia intensiva: revisão integrativa. Rev enferm UFPE on line.2015; 9(5):7957-66.

6. Moreira TM, Araújo TL, Pagliuca LMF. Alcance da teoria de King junto à família de pessoas portadoras de Hipertensão Arterial Sistêmica. Rev Gaúcha Enferm 2001; 22(2): 74-89.

7. Minayo MCS. 0 desafio do conhecimento: pesquisa qualitativa em saúde. 12. ed. São Paulo: Hucitec, 2010.

8. Pereira LACS et al. Educação em saúde com hipertensos analfabetos: relato de experiência. Rev Enferm UFPI. 2019; 8,Spec:77-80.

9. Bezerra ASM, Lopes JL, Barros ALBL. Adesão de pacientes hipertensos ao tratamento medicamentoso. Rev Bras Enferm. 2014 jul-ago;67(4):550-5.

10. Lima DBS et al Associação entre adesão ao tratamento e tipos de complicações cardiovasculares em pessoas com hipertensão arterial. Texto Contexto Enferm. 2016; 25(3): e0560015.

11. Sousa LSN et al. Conhecimento do enfermeiro sobre a prevenção do pé diabético: revisão integrativa da literatura. Revista Brasileira em Promoção da Saúde, 2017; 30(3):1-10.

12. Souza NPG et al. Conceptions of illness from hypertension and Diabetes Mellitus among a group of hospital inpatients. Rev. Enferm. UERJ, 2015; 23(1): 52-7.

13. Pereira NPA, Lanza FM, Viegas SMF. Vidas em tratamento para Hipertensão Arterial Sistêmica e Diabetes Mellitus: sentimentos e comportamentos. Rev Bras Enferm [Internet]. 2019;72(1):109-17.

14. Moretti CA, Dallegrave D, Riggo LJA, Dalberto EM. Implementação da consulta de enfermagem na Estratégia Saúde da Família: desafios e potencialidades. J Nurs Health. 2016;6(2):309-20.

15. Teixeira AK, Silva LFS, Silva ANC, Fernandes SSF, Oliveira ACAO, Freire EDAF et al. Cuidado clínico de enfermagem à pessoa com Úlcera Venosa fundamentado na teoria de Imogene King. REAID, 2019;88(26): 1-8.

16. King IM.A theory for nursing: systems, concepts, process. Tampa: Delmar; 1981. 17. Lima AF, Moreira ACA, Silva MJ, Monteiro PAA, Teixeira PG. A percepção do idoso com diabetes acerca de sua doença e o cuidado de enfermagem. Cienc Cuid Saude. 2016;15(3):522-9.

18. Silva KM, Vicente FR, Santos SMA. Consulta de enfermagem ao idoso na atenção primária à saúde: revisão integrativa da literatura. Rev Bras Geriatr Gerontol. 2014; 17 (3): 681-7.

19. Pereira IMO. Proposta de intervenção interdisciplinar para a adesão dos pacientes ao tratamento da hipertensão arterial sistêmica. Liph Science. 2015;2(2):21-40.

20. Platenik NC, Cruz TZ, Geller M. Abordagem terapêutica para o tratamento de hipertensão arterial sistêmica entre pacientes apresentando neurofibromatose tipo 1. Revista JOPIC. 2019; 4(2): 65-70. 